\title{
GekkoFS - A temporary burst buffer file system for HPC applications
}

\author{
Marc-André Vef ${ }^{1}$, Nafiseh Moti ${ }^{1}$, Tim Süß ${ }^{1}$, Markus Tacke ${ }^{1}$, Tommaso Tocci ${ }^{2}$, Ramon Nou ${ }^{2}$, Alberto Miranda ${ }^{2}$, \\ Toni Cortes ${ }^{23}$, André Brinkmann ${ }^{1}$ \\ ${ }^{1}$ Zentrum für Datenverarbeitung, Johannes Gutenberg University Mainz, 55128 Mainz, Germany \\ ${ }^{2}$ Computer Science Department, Barcelona Supercomputing Center, Barcelona 08034, Spain \\ ${ }^{3}$ Department of Computer Architecture, Universitat Politecnica de Catalunya, Barcelona 08034, Spain
}

E-mail: vef@uni-mainz.de; moti@uni-mainz.de; suesst@uni-mainz.de; tacke@uni-mainz.de; tommaso.tocci@bsc.es; ramon.nou@bsc.es; alberto.miranda@bsc.es; ramon.nou@bsc.es; toni.cortes@bsc.es; brinkman@uni-mainz.de

\begin{abstract}
Many scientific fields increasingly use High-Performance Computing (HPC) to process and analyze massive amounts of experimental data while storage systems in today's HPC environments have to cope with new access patterns. These patterns include many metadata operations, small I/O requests, or randomized file I/O, while general-purpose parallel file systems have been optimized for sequential shared access to large files.

Burst buffer file systems create a separate file system that applications can use to store temporary data. They aggregate node-local storage available within the compute nodes or use dedicated SSD clusters and offer a peak bandwidth higher than that of the backend parallel file system without interfering with it. However, burst buffer file systems typically offer many features that a scientific application, running in isolation for a limited amount of time, does not require.

We present GekkoFS, a temporary, highly-scalable file system which has been specifically optimized for the aforementioned use cases. GekkoFS provides relaxed POSIX semantics which only offers features which are actually required by most (not all) applications. GekkoFS is, therefore, able to provide scalable I/O performance and reaches millions of metadata operations already for a small number of nodes, significantly outperforming the capabilities of common parallel file systems.
\end{abstract}

Keywords Distributed File Systems, High-Performance Computing, Burst Buffers, POSIX

\section{Introduction}

High-Performance Computing (HPC) applications are currently significantly changing. Traditional HPC applications have been compute-bound, large-scale simulations, while today's HPC community is additionally moving towards the generation, processing, and analysis of massive amounts of experimental data. This trend, known as data-driven science, is affecting many different scientific fields, some of which have made significant progress tackling previously unaddressable challenges thanks to newly developed techniques $[1,2]$.

Most data-driven workloads are based on new algorithms and data structures like graph databases, which impose new requirements on HPC file systems $[3,4]$. Among others, they include large numbers of metadata operations, data synchronization, non-contiguous and random access patterns, and small $\mathrm{I} / \mathrm{O}$ requests $[3,5]$. These access patterns differ significantly from past workloads which mostly performed sequential I/O operations on large files. These new access patterns do not only slow down the data-driven applications themselves but can also heavily disrupt other applications that are concurrently accessing the shared storage system $[6,7]$. Consequently, conventional parallel file systems (PFS) cannot handle these workloads efficiently and data-driven applications suffer from prolonged I/O latencies, reduced throughput, and long waiting times.

Software-based approaches to support data-driven applications try to align the new access patterns to the capabilities of the underlying PFS. These approaches include application modifications or use middleware and high-level libraries (e.g., ADIOS [8], or HDF5 [9]). Adapting the software is typically time-consuming, dif- 
ficult to couple with big data and machine learning libraries, or sometimes (based on the underlying algorithms) just impossible.

Hardware-based approaches move from magnetic disks, which are still the main backend technology for PFSs, to NAND-based solid-state drives (SSDs). Nowadays, many supercomputers deploy SSDs, which provide a high sequential and random access perfor-

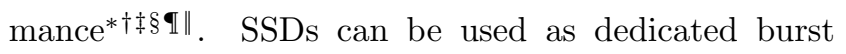
buffers [10] or as node-local burst buffers. To achieve high metadata performance, burst buffers can be deployed in combination with a dynamic burst buffer file system $[11,12]$.

Generally, burst buffer file systems increase performance compared to a PFS without modifying an application. Therefore, they typically support POSIX which provides the standard semantics accepted by most application developers. Nevertheless, enforcing POSIX can severely reduce a PFS's peak performance [13]. Further, many POSIX features are not required for most scientific applications [14], especially if they can exclusively access the file system. Similar argumentations hold for other advanced features like fault tolerance or security.

We present GekkoFS, a temporarily deployed, highly-scalable distributed file system for HPC applications. GekkoFS pools together fast node-local storage resources and provides a global namespace accessible by all participating nodes. It relaxes POSIX by removing some of the semantics that most impair I/O performance in a distributed context, and it takes previous studies on the behavior of HPC applications into ac-

\footnotetext{
*Aurora: http://aurora.alcf.anl.gov

${ }^{\dagger}$ Cori: $\quad$ http://www.nersc.gov/users/computationalsystems/cori

${ }^{\ddagger}$ Marenostrum: https://www.bsc.es/marenostrum/marenostrum

$\S$ Mogon II: https://hpc.uni-mainz.de

ISierra: https://computation.llnl.gov/computers/sierra

"Summit: https://www.olcf.ornl.gov
}

count [14] to heavily optimize the most used file system operations.

For load-balancing, all data and metadata are distributed across all nodes using the HPC RPC framework Mercury [15] in combination with the HPC threading framework Argobots [16, 17]. The file system runs in user-space and can be easily deployed in under 20 seconds on a 512 node cluster by any user. It, therefore, can be used in a number of temporary scenarios, e.g., during the lifetime of a compute job or in longer-term use cases, such as campaigns in which data is simultaneously accessed by many nodes in short bursts.

Besides being able to offer the combined storage capabilities of node-local storage devices, GekkoFS' goal is to accelerate I/O operations in common HPC workloads that are challenging for modern PFSs. We demonstrate how our lightweight, yet highly distributed file system can achieve scalable data and metadata performance, achieving tens of millions of metadata operations per second on a 512 node cluster. At the same time, GekkoFS is able to run complex applications, such as OpenFOAM solvers [18]. These features are achieved while GekkoFS operates synchronously and with a strong consistency model for file system operations that target a specific file or directory.

In this work, we built on the conference paper by the authors M.-A. Vef et al. [19] and extend it by a more detailed description of its architecture and core techniques in Section 3. Section 4 offers a deeper analysis of additional experiments with an in-depth evaluation of the file system's metadata and data performance on the MOGON II supercomputer. In Section 4.4, we evaluate GekkoFS' I/O variability on the MareNostrum IV supercomputer and compare it with the capabilities of its GPFS installation. Further, Section 4.5 investigates GekkoFS' and Lustre's effects on the network commu- 
nication when the user application OpenFOAM [18] is run. Finally, we conclude in Section 5 and discuss further research directions.

\section{Related work}

In this section, we give an overview over existing HPC file systems and discuss the differences to GekkoFS.

\section{General-purpose parallel file systems Most HPC} systems are equipped with a backend storage system which is globally accessible using a parallel file system (e.g., GPFS [20], Lustre [21,22], BeeGFS [23], or PVFS [24]). These file systems offer a POSIX-like interface, which allows applications to run as they were accessing a local file system, focusing on data consistency and long-term storage. However, due to the nature of the file system being globally accessible, single applications can disrupt the I/O performance of others applications as well. In addition, these file systems are not well suited for small file accesses, in particular on shared files, which can be found in scientific applications [3].

GekkoFS' design does not focus on long-term storage. Contrary to previously mentioned and permanently available file systems, it offers a separate namespace only accessible to nodes participating within the context of an HPC job or other temporary defined groups. When such a context is finished, all data is deleted. Further, GekkoFS offers a relaxed POSIX environment. As such, our file system is able to provide a significant increase in metadata performance and reduces the impact on other applications running on the same HPC system.

Burst buffers Burst buffers are fast, intermediate storage systems that aim to reduce the load on the global file system and on reducing an applications' I/O overhead [10]. Essentially, burst buffers can be categorized into two groups [11]: remote-shared and nodelocal. Remote-shared burst buffers, e.g., DDN's IME** and Cray's DataWarp ${ }^{\dagger \dagger}$, are centralized, dedicated I/O nodes structured as a forwarding layer.

Node-local burst buffers are generally collocated with the compute nodes and can be dependent on the PFS (e.g., PLFS [12]). In some cases, these burst buffers can also be managed directly by the PFS itself [25]. The Hermes [26] I/O middleware library provides a distributed I/O buffering system which transparently combines multi-tiered storage (e.g., node-local SSDs) and memory hierarchies of supercomputer environments. It uses multiple data placement techniques to place data on all storage layers efficiently and therefore considers both local and shared resources as burst buffers.

BurstFS [11], perhaps the most related work to ours, is a standalone burst buffer file system which does not require a centralized instance as well. However, GekkoFS is not limited to write data locally like BurstFS. Instead, all data is distributed across all participating file system nodes to balance data workloads for write and read operations without sacrificing scalability. BeeOND [23] can create a job-temporal file system on a number of nodes similar to GekkoFS. BeeOND is, in contrast to our file system, POSIX compliant and our GekkoFS' measurements show a much higher metadata throughput than offered by BeeOND (see Section 4.2).

POSIX The term Portable Operating System Interface $^{*}$ is rather broad and at times ambiguous. When

\footnotetext{
** IME: ddn.com/products/ime-flash-native-data-cache

††Datawarp: https://www.cray.com/datawarp

*http://pubs.opengroup.org/onlinepubs/9699919799/
} 
used within a file system context, POSIX is typically referred to as POSIX I/O, targeting the behavior of write and read operations. Henceforth, POSIX refers in this paper to POSIX I/O. POSIX was developed over 25 years ago for file accesses by a single process. Yet, until today there is no support for parallel $\mathrm{I} / \mathrm{O}$ which involves multiple I/O operations on a single shared file.

To provide POSIX semantics in a distributed environment with a strong consistency model and shared accesses, parallel file systems typically rely on expensive distributed locking mechanisms to avoid conflicts, such as byte-range locking (e.g., Lustre [21] and GPFS [20]). However, strong consistency semantics and poor parallel I/O support in file systems [27] combined with scientific applications' access patterns [3] are the main reasons for HPC I/O challenges. Despite everincreasing computing performance, data-intensive applications running on HPC systems face scalability challenges which prevents them to fully utilize the offered computing power [28].

One solution to reduce locking induced overhead is to give the responsibility that no shared conflicts occur to the application or external libraries. PVFS, e.g., does not implement a file system locking mechanism [24] [29]. GekkoFS supports this argumentation and does not provide a locking mechanism to avoid any global locking overhead.

Metadata scalability At its core, metadata in Unixlike operating systems can be categorized into three components: an objects metadata, a file's data, and a directory's contents [30]. Metadata is typically stored in an inode, containing information about the object's type (e.g., file, directory, symbolic links), its size, access permissions, and last modification time (mtime), for example. For data organization, file systems offer directories that store information about their contents in directory blocks.

However, inodes and directory blocks were not designed for parallel accesses because a single block can only be accessed by one process at a time. This is particularly relevant in distributed systems when a huge number of files is created in a single directory from multiple processes, a common workload in HPC environments $[12,25,31,32]$. In general, such systems distribute data across all available storage targets. While this technique works well for data, it does not achieve the same throughput when handling metadata $[33,34]$. This is caused by complex distributed locking of central data structures (generally managed by a metadata server instance) that are required to be accessed in parallel [31]. The file system community presented various techniques for handling metadata $[12,32,35,36,37,38]$, but this challenge is still prevailing and is becoming an even bigger challenge for upcoming data-science applications.

IndexFS [33], for example, is one such attempt to drastically improve metadata performance by using it as a middleware software on top of existing file systems, such as PVFS or Lustre. Similarly to GekkoFS, IndexFS uses a key-value database to store metadata information and it distributes metadata across multiple IndexFS servers. In addition, IndexFS uses various client caches to increase RPC efficiency further and assigns directories to IndexFS servers. GekkoFS, on the other hand, uses loosely coupled components and does not offer any caching functionality on clients. Instead, GekkoFS aggressively stripes all metadata across all servers. Moreover, in contrast to GekkoFS, IndexFS is still bound to implement POSIX file I/O semantics, requiring IndexFS to work with the file system protocols of the used underlying file system, e.g., pathname traversal or permission checking. GekkoFS relaxes POSIX and weakens the concept of directories 
which internally are no longer treated as mentioned directory blocks, directory entries, and inodes (see Section 3.5). In fact, GekkoFS entirely removes directory blocks and replaces directory entries by objects, stored within a strongly consistent key-value database which relinquishes the need for locking mechanisms within the file system. As a result, GekkoFS achieves tens of millions of metadata operations for 512 nodes and billions of files.

\section{Design and implementation}

In this section we introduce the goals and the design of GekkoFS. First, we present the goals of our file system. Next, we give a brief overview of the system components and the file system's architecture. Finally, we present details to each component of our system.

\subsection{Design goals}

We define the following goals for GekkoFS' design:

Functionality Any user should be able to deploy the file system on an arbitrary number of nodes without administrative assistance. The mount point of GekkoFS and the data directory, which the file system uses to store user data, is given when the file system is started. The mount point should then present the user with a single global namespace, consisting of the aggregated node-local storage of each node.

Scalability To benefit from current and future storage and network technologies, GekkoFS should scale with an arbitrary number of nodes and efficiently use available hardware.

Consistency model GekkoFS should provide the same consistency as POSIX for any file system operation that accesses a specific data file. This includes read and write operations as well as any metadata operations that target a single file, e.g., file creation. Nevertheless, consistency of directory operations, for instance, can be relaxed.

Fast deployment Compute time in HPC environments is valuable and expensive and should not be wasted for the purpose of file system deployment. Therefore, GekkoFS' startup should be finished within one minute to be used immediately by applications once the startup succeeds.

Hardware independence GekkoFS should be able to utilize all networking hardware that is commonly used in HPC clusters, such as Infiniband or Omni-Path, and fully support the native protocols of these fabrics to efficiently move data between file system nodes. In addition, GekkoFS should work with any modern and future storage subsystem that is (or will be) attached to compute nodes with the condition that the node-local storage is accessible at a path permitted to the user.

\subsection{Overview}

GekkoFS aims to offer a user-space file system for the lifetime of a particular use case, e.g., within the context of an HPC job. The file system uses the available local storage of compute nodes and combines their node-local storage into a single global namespace. For scalability and balanced usage of all available storage, data and metadata are distributed across all file system nodes.

Before an application is started, a file system server process is launched on each node with information about the file system mount point, the location where file system data is stored, and a list of participating nodes. An application uses the file system by preloading the GekkoFS client interposition library that intercepts file system operations on the GekkoFS mount point. 
The client library can also be used to stage-in or stageout data into GekkoFS from the PFS or vice versa, if necessary. For example, to copy data from a PFS path to a GekkoFS path, a user may use the cp command on the command line interface (CLI) while the interposition library is preloaded. When a GekkoFS operation is intercepted, the client forwards the operation to the responsible server, determined by hashing the file's path, where it is directly executed. To achieve a balanced data distribution for large files, each file is additionally split into equally sized chunks by the client and then distributed among the servers. Due to this communication scheme, there is no communication between server instances.

\subsection{POSIX relaxation}

From definitions on how I/O interfaces work, come certain requirements and expectations on how the file system retrieves results. The POSIX model inherently leads to a consistency model, which requires atomicity and locking mechanisms in a distributed environment. While local file systems are able to efficiently provide such a consistency model, it can result in scalability issues in a PFS (see Section 2).

The POSIX consistency is especially challenging for the scalability of a PFS in the following cases:

1. Atomic operations within a distributed environment that require exclusive write or read access to a central data structure. Such atomicity is typically achieved by acquiring a global lock on the desired data structure, which can impair concurrent work. Examples of such operations are file create(), since it involves modifying the parent directory and readdir() as it needs to create a snapshot of a directory's current state and may target an arbitrary number of file system objects.

2. Cache coherency protocols. While there are sev- eral advantages to provide various forms of caching in a PFS, it is generally not clear whether an application with particular semantics can benefit from such general caching protocols. Moreover, distributed cache coherency protocols often require a large body of network communication to keep synchronization, which is commonly impracticable at larger scales.

Therefore, similarly to PVFS [39] and OrangeFS [29], GekkoFS does not provide a global lock mechanism. In this sense, applications should be responsible to ensure that no conflicts occur, in particular, w.r.t. overlapping file regions, to avoid complex locking within the file system. However, the lack of distributed locking has consequences for operations where the number of affected file system objects is unknown a priori, e.g., when requesting the contents of a directory. In these indirect file system operations, GekkoFS does not guarantee to return the current state of the directory. In other words, readdir() operations which are called by the $1 \mathrm{~s}-1$ or $\mathrm{rm}-\mathrm{rf} / *$ commands, for instance, follow an eventual-consistency model. Also for the above-stated reasons, each operation in GekkoFS is synchronous without any form of caching within the context of file system operations. This not only reduces file system complexity but allows for an evaluation of GekkoFS' raw performance capabilities.

Moreover, studies on the behavior of HPC applications have shown that many features that file systems offer are rarely used or not used at all, such as move/rename operations [14]. Instead, these operations are typically only called from a console after the actual simulation ends and when parallel access is not required anymore. Taking these observations into account, GekkoFS' does not optimize towards move or rename operations and linking functionality although supporting it rudimentarily.

Finally, security management in the form of access 


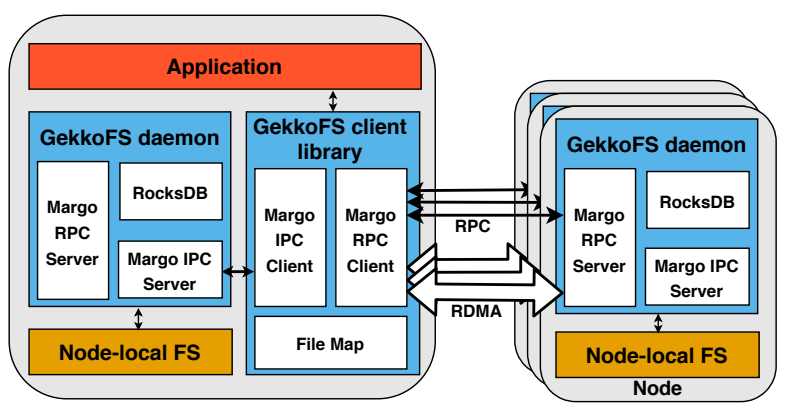

Fig.1. GekkoFS architecture with its components

permissions is not maintained by GekkoFS as it already implicitly follows the security protocols of the nodelocal file system that is used to store the file system's data and metadata. Data can only be staged into or out of GekkoFS if the user has the corresponding access rights on the backend file system.

\subsection{Architecture}

GekkoFS' architecture (see Figure 1) consists of two main components: a client library and a server process. An application that uses GekkoFS must first preload the client interposition library through the LD_PRELOAD environment variable which intercepts file system operations. However, because any function can be intercepted by the preloading library, all used I/O functions by an application must be reinterpreted by GekkoFS for the application to work. Therefore, the more complex an application the more functions need to be intercepted, potentially reimplementing a large percentage of the GNU C Library (glibc).

To limit the number of functions that need to be intercepted, GekkoFS uses the system call intercepting library ${ }^{\dagger}$ (syscall_intercept) which aims to solve this challenge by providing a low-level interface for hooking Linux system calls while still using the LD_PRELOAD method. As a result, the GekkoFS client only needs to intercept system calls, such as sys_mknod or sys_write,

\footnotetext{
${ }^{\dagger}$ https://github.com/pmem/syscall_intercept
}

while leaving the functionality of glibc, for instance, untouched.

Once the client has intercepted an I/O call, it forwards the I/O call to a server (GekkoFS daemon), if required. The GekkoFS daemon, which runs on each file system node, receives forwarded file system operations from clients and processes them, sending a response when finished. The daemons operate independently and do not communicate with other server processes on remote nodes, therefore being effectively unaware from each other. In the following paragraphs, we describe the client and daemon in detail.

GekkoFS client The client consists of three components: 1. An interception interface that catches relevant calls to GekkoFS and forwards unrelated calls to the node-local file system; 2. a file map that manages the file descriptors of opened files and directories, independently of the kernel; and 3. an RPC-based communication layer that forwards file system requests to local/remote GekkoFS daemons.

When the client library is first invoked by an application, it requests basic information about the mount point and the participating nodes from the local GekkoFS daemon. The interception library then checks for each file system operation if the GekkoFS mount point is used. If this is not the case, the call is passed through to the underlying file system. Whenever a file is opened, a new file map entry is created which associates the file handle with its path, among other information. Upon closing the file this entry is removed.

While a file is open, the client uses the file path $p$ for each related file system operation to determine the GekkoFS daemon node that should process it. Specifically, the path $p$ is hashed using a hash function $h$ to resolve the responsible daemon for an operation by 
calculating:

$$
\text { nodeID }=h(p) \quad(\bmod \text { number of GekkoFS nodes })
$$

The corresponding operation is then forwarded via an RPC message to the daemon with the unique nodeID where it is directly executed. In other words, GekkoFS uses a pseudo-random distribution to spread data and metadata across all nodes, also known as wide-striping. Because each client is able to independently resolve the responsible node for a file system operation, GekkoFS does not require central data structures that keep track of where metadata or data is located.

In addition, to achieve a balanced data distribution for large files, data requests are split into equally sized chunks before they are distributed across file system nodes. During data transfers between client and GekkoFS daemons, the client exposes the relevant chunk memory region to the daemon which accesses it via remote-direct-memory-access (RDMA), if supported by the underlying network fabric protocol.

GekkoFS daemon A GekkoFS daemon's purpose is to process forwarded file system operations of clients to store and retrieve data and metadata that hashes to a daemon. To achieve this goal, GekkoFS daemons consist of three parts: 1. A key-value store (KV store) used for handling metadata operations, 2. an I/O persistence layer that reads/writes data from/to the underlying node-local storage system, and 3. an RPC-based communication layer that accepts local and remote connections to handle file system operations.

Each daemon operates a single local RocksDB keyvalue store which provides a high-performance embedded database for key-value data, based on a logstructured merge-tree (LSM) [40]. RocksDB is optimized for NAND storage technologies with low latencies and thus fits GekkoFS' needs as SSDs are primarily used as node-local storage in today's HPC clusters.
For the communication layer, we leverage on the Mercury RPC framework, which allows GekkoFS to be network-independent, achieving one of our design goals (see Section 3.1). Mercury is an RPC communication library developed by the Argonne National Laboratories (ANL), which focuses on HPC environments [15]. In contrast to other RPC frameworks, Mercury is able to use the native network transport layer and can, therefore, handle large data transfers efficiently. Mercury's Network Abstraction Layer, which provides a high-level interface on top of the lower level network fabrics, offers a wide variety of plugins to natively support common fabric protocols, e.g., InfiniBand or Omni-Path. When available, large data transfers are processed via RDMA or cross-memory attach (CMA) in remote and local communications, respectively. This allows GekkoFS to efficiently transfer data within the file system.

Within a GekkoFS, Mercury is interfaced indirectly through the Margo library which provides Argobotsaware wrappers to Mercury's API with the goal to provide a simple multi-threaded execution model [17]. Argobots is a lightweight low-level threading and tasking framework, developed to support massive on-node concurrency in modern HPC environments [16]. Using Margo allows GekkoFS daemons to minimize resource consumption of Margo's progress threads and handlers, that accept and handle RPC requests [17].

\subsection{Rethinking metadata management}

Modern distributed storage systems typically employ several strategies to distribute metadata and data across all available storage targets [20, 21, 23]. As described in Section 2 this technique usually works well for data but does not achieve the same efficiency and performance (throughput or operations per second) when handling metadata due to expensive distributed locking mechanisms. Based on these observations, we aim at 
forming a file system that performs well for any type of direct metadata operations and allows them to scale to an arbitrary number of nodes. We present our methodology in two steps targeting metadata management and its contents.

Decoupled wide-striping We aim to achieve metadata scalability by decoupling directory contents from directory blocks, allowing GekkoFS to operate without a global lock manager, and by wide-striping metadata across all file system nodes. Instead of using directory blocks which are challenging to use in distributed environments, each directory entry is stored in a daemon's $\mathrm{KV}$ store where the file path is used as the key and the value contains the file's metadata. As a result, each file becomes individually accessible by its path, resulting in a flat namespace where paths that share the same prefix are considered as the children of a directory. Finally, by using the previously described distribution algorithm (see Sec. 3.4), metadata entries are striped across all nodes.

However, using the path of a file system object as an index within a flat namespace comes not without cost. If a directory is, e.g., moved to a different file system path, the paths of all its contents would have to be recursively modified as well. Depending on the size of the directory and due to the distribution of metadata, this can be a time-consuming process, as metadata might need to be migrated if the hashes for the new paths lead to different file system nodes. Nonetheless, as described in Section 3.3, GekkoFS explicitly disallows such operations, since they are rarely used in HPC applications.

Metadata contents A file system that is only temporarily accessed during a specific use case, e.g., within the context of an HPC job, may not require exposing all metadata fields to an application. First, we categorize metadata fields into three categories: redundant, rarely used, and mandatory. The first category includes permission bits, user id, and group id information, which are used for security purposes, but that we consider as redundant as GekkoFS has to follow the node-local file system's security protocols (see Section 3.3). The second category contains metadata fields which are rarely used by HPC applications, including timestamps, the inode number, and block size information. The metadata fields of the first two categories are disabled by default to save space within the KV store and to reduce the amount of metadata that is send over the network. However, they can still be enabled if needed.

The third category contains mandatory metadata fields which cannot be turned off: the file type and the file size. Although we do not use traditional directories which store all entries in a directory block, we still support directory types because applications often check for a directory's existence before it is populated. The second mandatory metadata field, the file size, is used to keep track of a file's data boundaries. This is particularly of interest when working with a sparse file in which file systems generally do not write regions to disk that contain no information. In other words, the file size is used to differentiate between an application accessing outside a file's dimensions or within a sparse region of the file.

\subsection{Data management}

GekkoFS manages data by utilizing a compute node's local storage system. In the following paragraphs, we describe GekkoFS' I/O protocol and explain how shared file access is managed in an environment where global locks are not used.

I/O protocol Similarly to metadata, data (split into equally sized chunks) is evenly distributed across all 


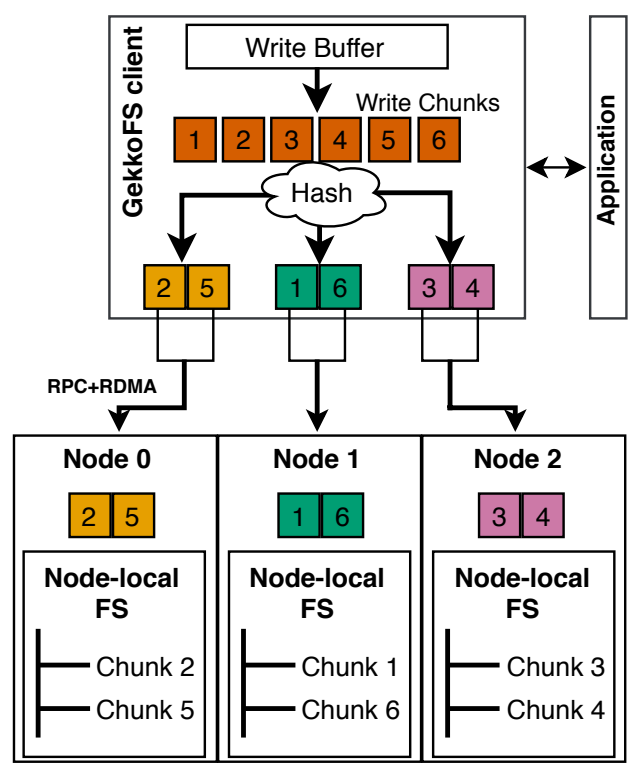

Fig.2. GekkoFS' write operation where a write buffer is split into six chunks and then distributed among three daemons. Each daemons stores its chunk in a node-local file system.

file system nodes. Within a node-local file system, each chunk is represented as a file and is named after the chunk's numeric identifier, which also describes the chunk's data offset. For example, in a 1 MiB chunksized file system, the chunk number 2 references a file's offset starting at address $2 \mathrm{MiB}$. Chunks of the same file in GekkoFS are stored within the scope of the same directory in the node-local file system.

Figure 2 shows a write operation from the clients point of view. In the given case, the write buffer is split into six chunks (dependent on the chunk size). For each chunk, GekkoFS computes the target node with the help of the file's path and chunk identifier, grouping chunks that target the same node. The client then sends an RPC message to each target daemon node, each independently handling the write request for a group of chunks. Each GekkoFS daemon accesses the client's memory via RDMA and writes the corresponding chunks to its node-local file system. If the target daemon refers to the local machine, data is moved via CMA, which allows accessing a set memory region of another process on the same machine without copying it. In the event of serving multiple chunks per daemon, data transfer and disk I/O may be done in parallel. The read operation works similarly but in reverse. As explained in Section 3.3, all I/O operations are synchronous without any caching mechanisms on clients or daemons, while the caching mechanisms of the local file systems are used.

Shared write conflicts As shown in Section 3.3, GekkoFS does not implement a global locking manager. This can impose challenges when working with shared files. For instance, when two or more processes write to the same file region at the same time they could cause a shared write conflict, resulting in an undefined behavior with regards to the data which is written to the underlying storage device. However, because of GekkoFS' decoupled design, the above-described locking conflicts can, in fact, be handled by any file system daemon locally. This is owing to the use of a POSIX-compliant node-local file system which stores the corresponding data chunks and, in the case of a shared write conflict, serializes access to the same chunk file. Moreover, because a file may be distributed across many chunk files and nodes, multiple conflicts in the same file only affect one chunk at a time. Hence, other chunks of that file are not disrupted during such a potential shared write conflict.

\section{Evaluation}

In this section, we evaluate the performance of GekkoFS based on various microbenchmarks which catch access patterns that are common in HPC applications. First, we describe the experimental setup and introduce the workloads that we simulate with microbenchmark applications. Then, we investigate GekkoFS' startup time and compare GekkoFS' meta- 
data performance against a Lustre parallel file system. Although GekkoFS and Lustre have different goals, we point out the performances that can be gained by using GekkoFS as a burst buffer file system. In addition, we compare GekkoFS' metadata performance with BeeGFS' BeeOND burst buffer file system which is used similarly as GekkoFS. Then, we evaluate GekkoFS' data performance and compare them with BeeGFS' BeeOND and discuss the measured results. Further, we investigate GekkoFS' I/O variability and worst-case performance Finally, we explore GekkoFS' with Lustre's effects on the network when the OpenFOAM application is used.

\subsection{Experimental setup}

We used three supercomputers in our experiments: MOGON II at the Johannes Gutenberg University Mainz in Germany ${ }^{\ddagger}$, MareNostrum IV at the Barcelona Supercomputing Center in Spain ${ }^{\S}$, and the NEXTGenIO prototype $\mathbb{I}$.

MOGON II consists of 1,876 nodes in total, with 822 nodes using Intel 2630v4 Intel Broadwell processors (two sockets each) and 1046 nodes using Xeon Gold 6130 Intel Skylake processors (four sockets each). If not otherwise noted, Intel Broadwell processors are used in all presented experiments. The node main memory capacity ranges from $64 \mathrm{GiB}$ up to $512 \mathrm{GiB}$ of memory.

The cluster uses $100 \mathrm{Gbit} / \mathrm{s}$ Intel Omni-Path interconnect to establish a fat-tree between all compute nodes and offers a 7.5 PiB storage backend, managed by multiple Lustre parallel file systems. In addition, each node provides a data center Intel SATA SSD DC S3700 Series with $200 \mathrm{GiB}$ or $400 \mathrm{GiB}$ of available storage as a scratch-space (XFS formatted) usable within

\footnotetext{
${ }^{\ddagger}$ Mogon II: https://hpc.uni-mainz.de

$\S$ Marenostrum: https://www.bsc.es/marenostrum/marenostrum

INEXTGenIO prototype: http://www.nextgenio.eu/
}

a compute job. In our experiments, we use these SSDs for storing data and metadata of GekkoFS or BeeGFS. Both GekkoFS and BeeGFS use internal chunk sizes of $512 \mathrm{KiB}$.

All Lustre experiments were performed on a Lustre scratch file system with 12 Object Storage Targets (OSTs), 2 Object Storage Servers (OSSs), and 1 Metadata Service (MDS) with a total of 1.2 PiB of storage.

For GekkoFS and BeeGFS experiments, all SSD contents are removed, and all kernel caches, i.e., buffer, inode, and dentry caches are flushed before each experiment iteration. In addition, the GekkoFS daemons are restarted (requiring less than 20 seconds for 512 nodes) before each experiment iteration as well. The GekkoFS daemon and the BeeGFS storage and metadata services, and the application under test are pinned to separate processor sockets to ensure that file system and application do not interfere with each other.

MareNostrum IV uses 3,456 Lenovo ThinkSystem SD530 compute nodes on 48 racks. Each node uses two Intel Xeon Platinum $816024 \mathrm{C}$ chips with 24 processors each at $2.1 \mathrm{GHz}$ which totals to 165,888 processes and 390 TB of main memory. In addition, each node provides an Intel SSD DC S3520 Series with 240 GiB of available storage, usable within a compute job. All GekkoFS experiments use these SSDs as their underlying storage.

A 100 Gb Intel Omni-Path Full-Fat Tree is used for the interconnection network connected to a total 14 PB of storage capacity offered by IBM's GPFS. All GPFS experiments where run in the projects file system which offers 4.4 PB of storage with an $8 \mathrm{MB}$ set file system block size. 
NEXTGenIO prototype is composed of 34 compute nodes" ${ }^{\|}$Each node has a dual Intel Xeon Platinum 8260M CPU @ $2.40 \mathrm{GHz}$ (i.e., 48 cores per node), 192 GiB of main memory and 3 TBytes of node-local Intel DCPMM memory. All GekkoFS experiments use these DCPMM memories as their underlying storage.

Compute nodes are interconnected with an OmniPath fabric with two fabrics per node, henceforward called ib0 and ib1, and they have a 56 Gbps InfiniBand to communicate with a Lustre server with 6 OSTs. GekkoFS uses TCP/IP over Omni-Path (emulated TCP) while Lustre uses the Omni-Path fabric with Infiniband emulation.

\subsection{Metadata performance}

On MOGON II, we simulated common metadata intensive HPC workloads using the mdtest ${ }^{* *}$ microbenchmark to evaluate GekkoFS' metadata performance. In our experiments, mdtest performs create, stat, and remove operations in parallel in a single directory. In particular, concurrent metadata operations in a single directory are an important workload in many HPC applications and are among the most difficult workloads for a general-purpose PFS to handle efficiently [31].

Each operation was performed on GekkoFS using 100,000 zero-byte files per process with 16 processes used per node. We chose the high number of files to force RocksDB to flush its in-memory tables to the underlying SSD to show RocksDB's consistent performance. As a result, a number of static sorted table files (sst files) were created on the backend SSD storage during our experiments.

All files created in the GekkoFS experiments are stored from the user application's perspective within a single directory. However, due to the flat namespace

\footnotetext{
$\|$ http://www.nextgenio.eu/

**https://github.com/hpc/ior
}

that is kept internally in the KV stores, there is conceptually no difference in which directory files are created. This is in contrast to a traditional PFS that may perform better if the workload is distributed among many directories instead of in a single directory. This is because inserting files into a directory is mostly sequentialized as multiple process cannot access the same file system block in parallel (see Section 2). As a result, application developers are often asked to create files in a separate directory per process, even if this does not fit their natural ordering.

Figure 3 compares GekkoFS with Lustre in three scenarios with up to 512 nodes: file creation, file stat, and file removal. The y-axis depicts the corresponding operations per second that were achieved for a particular workload on a logarithmic scale. During the experiments GekkoFS was running exclusively on the cluster without other compute jobs interfering. Each experiment was run at least five times with each data point representing the mean of all iterations. GekkoFS' workload scaled with 100,000 files per process, while Lustre's workload was fixed to four million files for all experiments. We fixed the number of files for Lustre's metadata experiments because Lustre was detecting hanging nodes when scaling to too many files.

Lustre experiments were run in two configurations: All processes operated in a single directory (single dir) or each process worked in its own directory (unique dir). Moreover, Lustre's metadata performance was evaluated while the system was accessible by other applications as well.

As seen in Figure 3, GekkoFS outperforms Lustre by a large margin in all scenarios, regardless of whether Lustre processes operated in a single or in an isolated directory. Compared to Lustre, GekkoFS achieved around 46 million creates per second $(\sim 1,405 \mathrm{x}), 44$ million stats per second $(\sim 359 \mathrm{x})$, and 22 million removes 


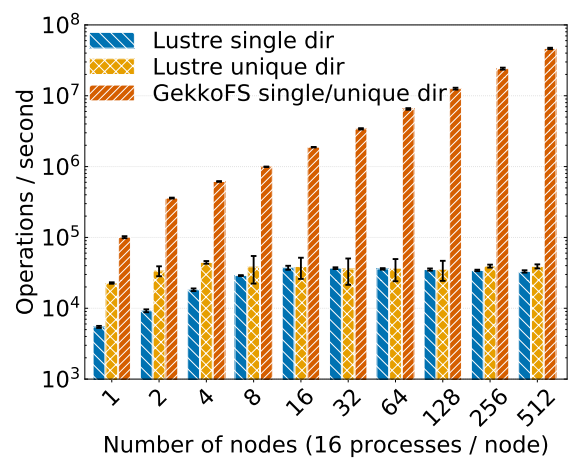

(a) Create throughput

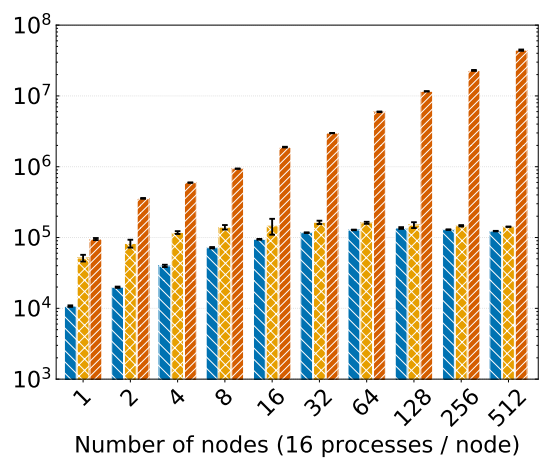

(b) Stat throughput

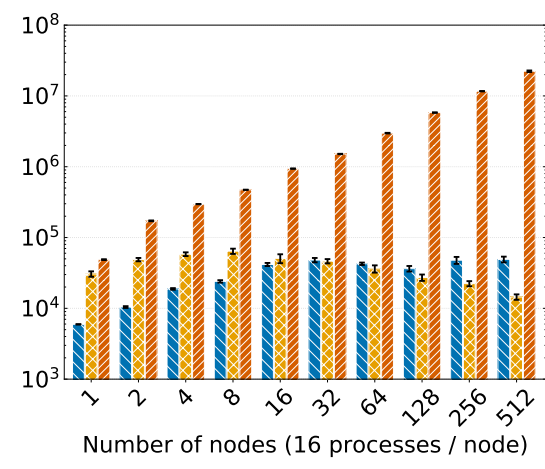

(c) Remove throughput

Fig.3. GekkoFS' file create, stat, and remove throughput for an increasing number of nodes compared to a Lustre parallel file system.

per second $(\sim 453 \mathrm{x})$ at 512 nodes. The standard deviation was computed as the percentage of the mean which, in all GekkoFS cases, was less than 3.5\%. For GekkoFS, each operation was performed synchronously without any caching mechanisms in place and shows close to linear scaling. Therefore, we achieve our scalability goal, demonstrating the performance benefits of distributing metadata and decoupling directory entries from non-scalable directory blocks (see Section 3.5).

The GekkoFS experiments were also run while Mogon II was used by other users during production, revealing network interference within the cluster. Up to 128 nodes we were unable to measure a difference in metadata operation throughput outside of the margin for error compared to the experiments in an undisturbed environment. For 256 and 512, we measured a reduced metadata operation throughput between $10 \%$ and $20 \%$ for create and stat operations. Remove operation throughput remained unaffected.

Lustre's metadata performance did not scale beyond approximately 32 nodes, demonstrating the aforementioned metadata scalability challenges in such a general-purpose PFS. Moreover, processes in Lustre experiments that operated within their own directory achieved a higher performance in most cases, except for the remove case where Lustre's unique dir remove throughput is reduced by over $70 \%$ at 512 nodes com- pared to Lustre's single dir throughput. This is because the time required to remove the directory of each process (in which it creates its workload) is included in the remove throughput and the number of created unique directories increases with the number of used processes in an experiment. Similarly, the time to create the process directories is also included in the create throughput but does not show the same behavior as the remove throughput, indicating optimizations towards create operations.

Figure 4 compares GekkoFS with a POSIXcompliant burst buffer file system: BeeGFS' BeeOND. Similar to GekkoFS, we configured BeeGFS to distribute metadata across all file system nodes. All experiments were run in two configurations: All processes operated in a single directory (single dir) or each process worked in its own directory (unique dir). GekkoFS' workload and all BeeGFS unique dir experiments were weakly scaled with 100,000 files per process. The workload for BeeGFS single dir experiments, on the other hand, was fixed to four million files. This is because BeeGFS' distribution to multiple metadata servers is coupled with the number of directories. Hence, operations in a single directory are only utilizing a single metadata server which inherently causes congestion on the corresponding node, affecting scalability. 


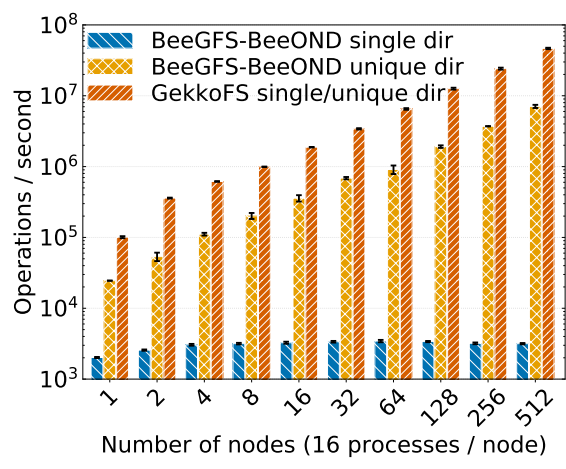

(a) Create throughput

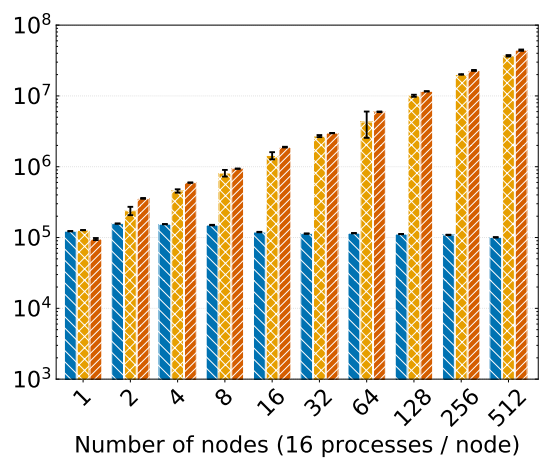

(b) Stat throughput

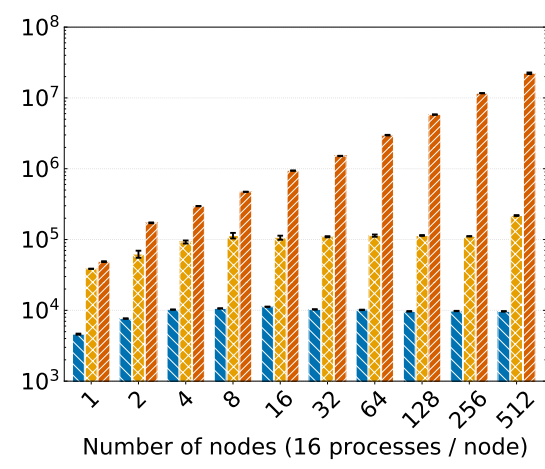

(c) Remove throughput

Fig.4. GekkoFS' file create, stat, and remove throughput for an increasing number of nodes compared to a BeeGFS' BeeOND.

Further, BeeGFS is equivalently deployed to GekkoFS, that is, BeeGFS is deployed in an ad hoc fashion for the lifetime of a compute job and destroyed afterwards while all metadata is stored on the node-local SSD. It is therefore only accessible by the benchmark application.

As seen in Figure 4, both GekkoFS' and BeeGFS' unique dir experiments show close to linear scalability with the number of nodes for create and stat operations. BeeGFS' remove operations, however, do not scale beyond four nodes, although throughput doubled from 256 to 512 nodes. GekkoFS' unique dir and single dir performance is equivalent as both scenarios are internally treated indifferently (see Section 3.5). In summary, at 512 nodes, GekkoFS achieved a $\sim 6.5 \mathrm{x}$ higher create throughput, a $\sim 1.2 \mathrm{x}$ higher stat throughput, and a $\sim 102 \mathrm{x}$ higher remove throughput, compared to BeeGFS unique dir scenario. All BeeGFS single dir experiments did not scale beyond four nodes due to above described limitations.

\subsection{Data performance}

On MOGON II, we evaluated GekkoFS' I/O performance with experiments that were designed to reflect some of the most difficult I/O patterns that scientific applications request from the PFS, such as small I/O requests or random access patterns. We used the $I O R^{\dagger \dagger}$

\footnotetext{
${ }^{\dagger \dagger}$ https://github.com/hpc/ior
}

microbenchmark which offers a rich configuration interface for evaluating a file system's I/O performance in various scenarios. We performed experiments with sequential and random access patterns in two configurations: Each process is accessing its own file (file-perprocess) and all processes access a single file (sharedfile).

We used four different write and read sizes for each configuration, in the following called transfer sizes: $8 \mathrm{KiB}, 64 \mathrm{KiB}, 1 \mathrm{MiB}$, and $64 \mathrm{MiB}$ to assess the performances for many small I/O accesses as well as for few large $\mathrm{I} / \mathrm{O}$ requests. In all experiments 16 processes ran on each client with each process writing and reading $4 \mathrm{GiB}$ in total.

GekkoFS data performance is not compared with the Lustre scratch file system as its peak performance, around $12 \mathrm{GiB}$ per second, is already reached for $\leq$ 10 nodes for sequential I/O patterns. Moreover, Lustre has shown to scale linearly for sequential access patterns in larger deployments with more OSSs and OSTs being available [41]. Instead, we first focus on GekkoFS' behavior with various transfer sizes and I/O patterns with the goal to achieve close to linear scalability, and then compare GekkoFS' I/O performance with BeeGFS' BeeOND where both file systems write and read data on the node-local SSDs.

We first evaluate file-per-process throughput perfor- 


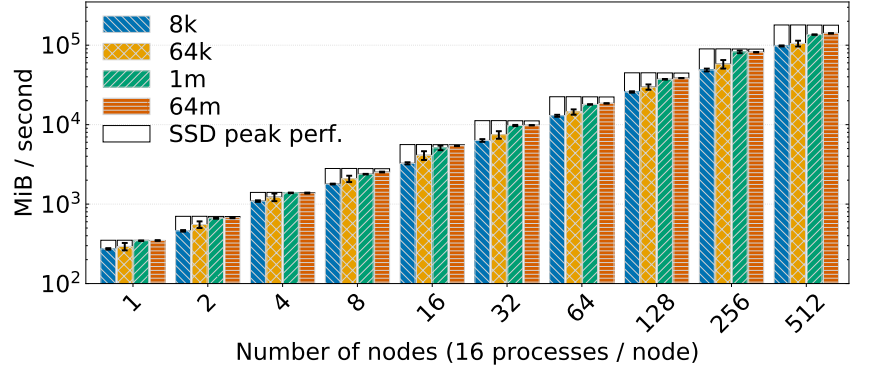

(a) Write throughput

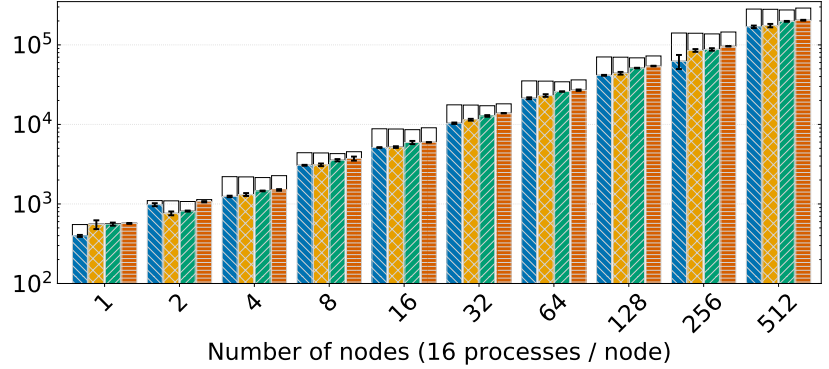

(b) Read throughput

Fig.5. GekkoFS' sequential throughput for each process operating on its own file compared to the plain SSD peak throughput.

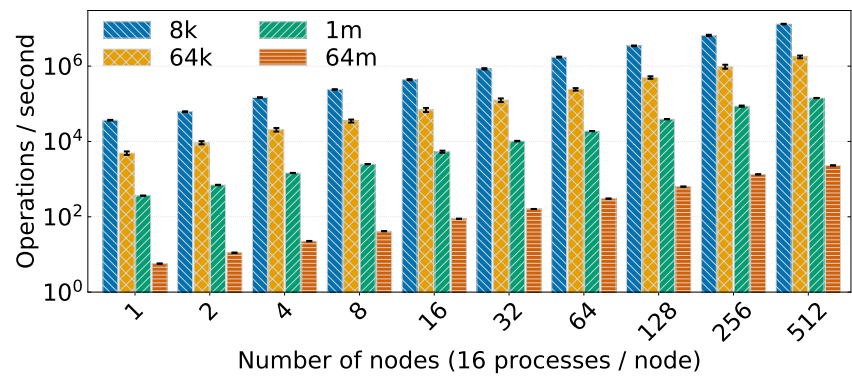

(a) Write IOPS

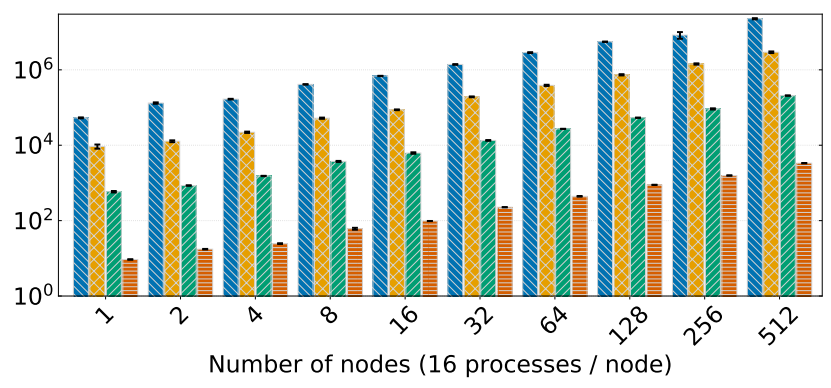

(b) Read IOPS

Fig.6. GekkoFS' sequential write and read operations per second for each process operating on its own file.

mances, write and read operations per second (IOPS), and latencies. Then we discuss shared-file performance and its challenges.

File-per-process and sequential access patterns: Figure 5 shows GekkoFS' sequential read and write throughput in $\mathrm{MiB} / \mathrm{s}$ for an increasing number of nodes for different transfer sizes. Each data point represents the mean of at least five iterations. The standard deviation, calculated as the percentage of the mean was smaller than $5 \%$ in all cases, except for $64 \mathrm{KiB}$ writes which varied up to $13 \%$. Further, each data point is compared to the peak performance that all aggregated SSDs could deliver for a given node configuration, visualized as a white rectangle, indicating GekkoFS' SSD usage efficiency.

In general, every result demonstrates GekkoFS' close to linear scalability, achieving about $141 \mathrm{GiB} / \mathrm{s}$ ( $~ 80 \%$ of the aggregated SSD peak bandwidth) and $204 \mathrm{GiB} / \mathrm{s}$ ( $\sim 70 \%$ of the aggregated SSD peak band- width) in respective write and read operations for $64 \mathrm{MiB}$ transfer sizes for 512 nodes.

Any I/O operation which is larger than the used chunk size of $512 \mathrm{KiB}$ will internally be split into equally sized chunk files on the node-local file system on one or more nodes (see Section 3.6). Chunks that hash to the same node are packaged together into the same $\mathrm{RPC}$ request, resulting in parallel RDMA accesses to the client's memory from multiple nodes. Therefore, chunks of $64 \mathrm{MiB} \mathrm{I} / \mathrm{O}$ requests can be served in parallel, achieving a higher throughput than $1 \mathrm{MiB}$ I/O requests. I/O requests smaller than the chunk size always target a single chunk, hence, a single node.

Figure 6 shows the $I / O$ operations per second (IOPS) for an increasing number of nodes (x-axis). GekkoFS achieved more than 13 million write IOPS and more than 22 million read IOPS at 512 nodes with an $8 \mathrm{KiB}$ transfer size. In general, the smaller the transfer size the higher the IOPS in all cases. This is because 


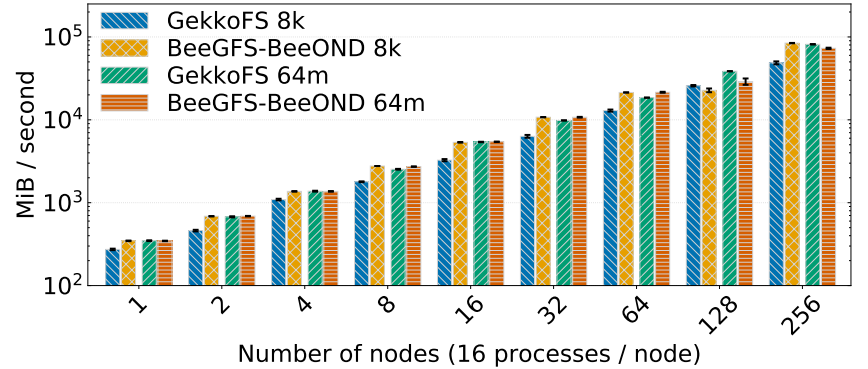

(a) Write throughput

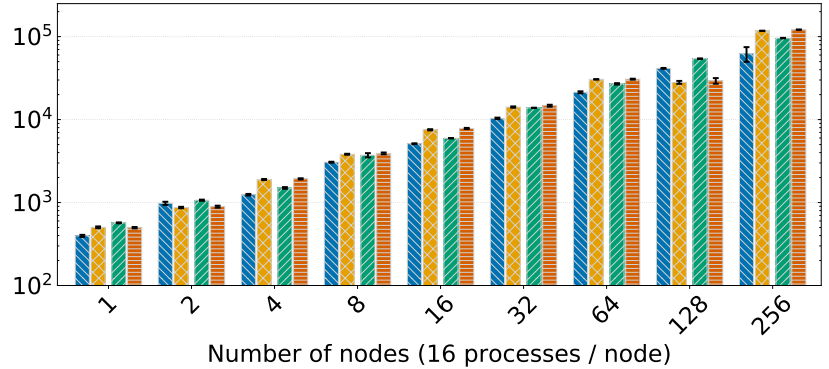

(b) Read throughput

Fig.7. GekkoFS' sequential throughput for each process operating on its own file compared BeeGFS' BeeOND.

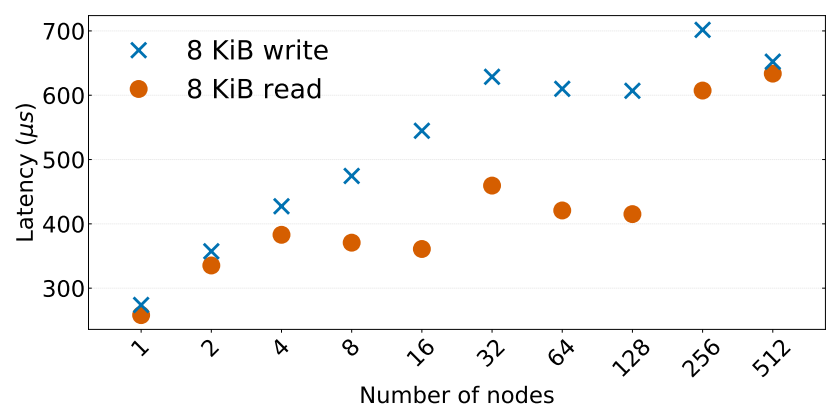

Fig.8. GekkoFS' I/O latencies for $8 \mathrm{KiB}$ transfer sizes.

small I/O requests reduce the amount of time the target node spends during an I/O operation of a chunk file. Therefore, the latency of each I/O request becomes the predominant factor in such an operation causing a decrease in throughput and an increase in IOPS.

Figure 8 depicts the I/O latencies for such small write and read requests, exemplarily shown for $8 \mathrm{KiB}$ transfer sizes. Each data point represents the mean latency for all write or read requests over five iterations. Because the experiments were weakly scaled, an increasing number of nodes translates into more I/Os. For instance, at 512 nodes the I/O latency of over 167 million operations were taken into account. In all cases, the I/O latencies were well below the capabilities of modern hard disk drives with access times of several milliseconds, showing GekkoFS' efficient utilization of the SSD storage backend. Note that the latencies of read operations are lower than of write operations as the latter involves an additional communication step to update the size of the file.

Further, we noticed rare but severe outliers which resulted in a high standard deviation that needs to be further investigated. Nonetheless, despite these outliers, the throughput and IOPS in various scenarios, as shown above, was stable with a low standard deviation.

Figure 7 compares GekkoFS with BeeGFS' BeeOND up to 256 nodes in two configurations: $64 \mathrm{MiB}$ and $8 \mathrm{KiB}$ transfer size to evaluate large and small transfer sizes alike. In both configurations, BeeGFS scales almost linearly with a standard deviation smaller than $9 \%$ in all cases. Similar to GekkoFS, BeeGFS used node-local SSDs for storage with the same workload, and it distributed all data across all available nodes with the test directory's stripe size is set to -1 . At 256 nodes with $64 \mathrm{MiB}$ transfer sizes, GekkoFS' write throughput is $\sim 1.12 \mathrm{x}$ higher than of BeeGFS while BeeGFS' read throughput is $\sim 1.26 \mathrm{x}$ higher than of GekkoFS. For 8 KiB transfer sizes, on the other hand, BeeGFS' write and read throughput is $\sim 1.73 \mathrm{x}$ and $\sim 1.86 \mathrm{x}$ higher than GekkoFS, respectively. Further experiments with BeeGFS and used transfer sizes ranging from $1 \mathrm{MiB}$ to $8 \mathrm{KiB}$ showed that write and read throughput remained similar, suggesting caching mechanisms for such transfer sizes. Since GekkoFS is not utilizing any caching mechanisms, a reduction in I/O throughput for smaller transfer sizes is expected as each $\mathrm{I} / \mathrm{O}$ request is sent individually and therefore becoming 


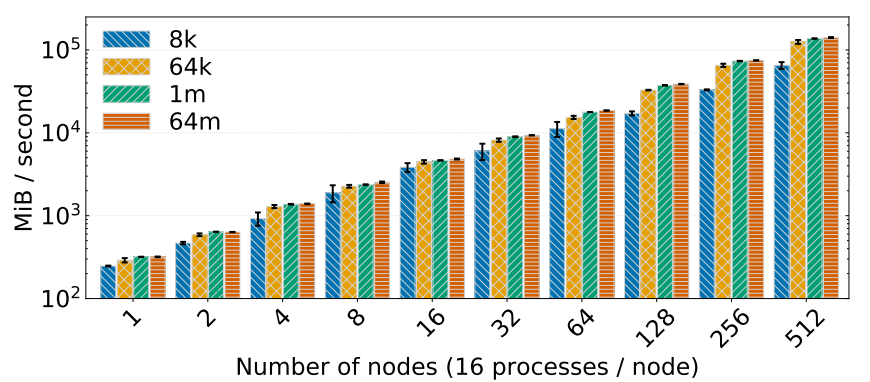

(a) Write throughput

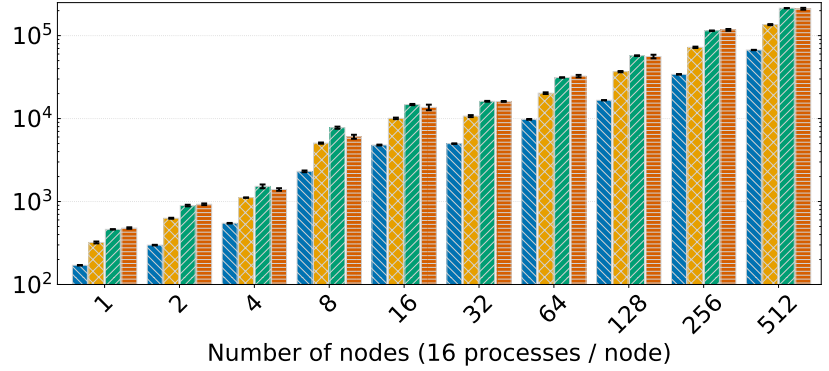

(b) Read throughput

Fig.9. GekkoFS' random write and read throughput for each process operating on its own file.

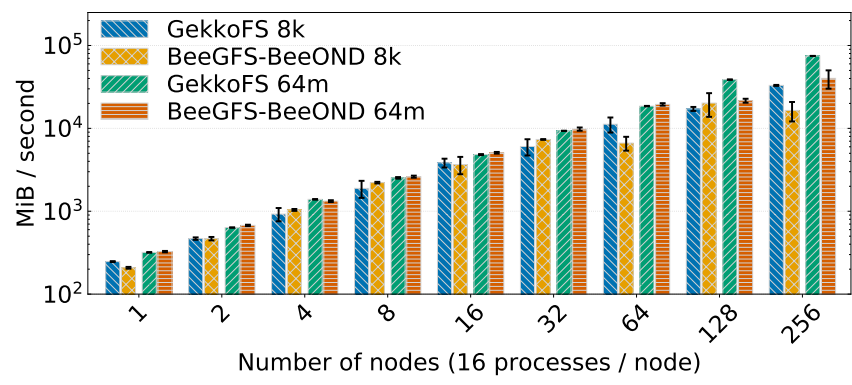

(a) Write throughput

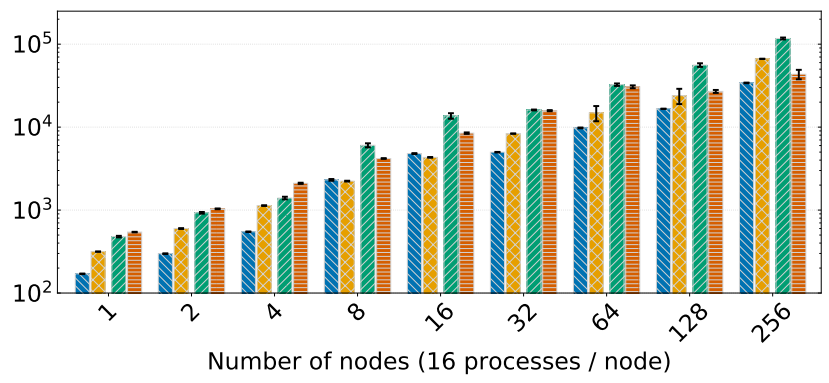

(b) Read throughput

Fig.10. GekkoFS' random write and read throughput for each process operating on its own file compared to BeeGFS' BeeOND.

increasingly more latency dependent in the process.

File per process and random access patterns:

Figure 9 shows GekkoFS' throughput for random accesses for an increasing number of nodes, showing close to linear scalability in all cases. The file system achieved up to $141 \mathrm{GiB} / \mathrm{s}$ write throughput and up to $204 \mathrm{GiB} / \mathrm{s}$ read throughput for $64 \mathrm{MiB}$ transfer sizes at 512 nodes.

The throughput for $64 \mathrm{MiB}$ and $1 \mathrm{MiB}$ transfer sizes are comparable to the sequential results. Nevertheless, write and read throughput decreased by approximately $33 \%$ and $60 \%$ for 512 nodes and a transfer size of $8 \mathrm{KiB}$. The reason is that transfer sizes larger than the chunk size internally access whole chunks while smaller transfer sizes access one chunk at a random offset.

Consequently, random accesses for large transfer sizes are conceptually the same as sequential accesses. In both cases whole chunks are written or read, resulting in a similar performance. Small transfer sizes, on the other hand, are slower than sequential accesses due to the resulting random access to chunks. Hence, applications may benefit from choosing smaller chunk sizes if their transfer sizes are small.

Figure 10 compares GekkoFS' throughput for random accesses with BeeGFS with up to 256 nodes. At 256 nodes with $64 \mathrm{MiB}$ transfer sizes, GekkoFS' write and read throughput is $\sim 1.86 \mathrm{x}$ and $\sim 2.7 \mathrm{x}$ higher than of BeeGFS, respectively, potentially showing the abovedescribed benefits of transfer sizes larger than the chunk size. For 8 KiB transfer sizes, GekkoFS' write throughput is $\sim 2 \mathrm{x}$ higher than of BeeGFS while BeeGFS' read throughput is $\sim 1.95 \mathrm{x}$ higher than of GekkoFS.

Single shared file: Shared file operations have many similarities to the previously presented experiments in which each process operated on its own file. For instance, in cases where the transfer size is bigger than the chunk size, each chunk file is only accessed by a single process, regardless of whether the whole file 


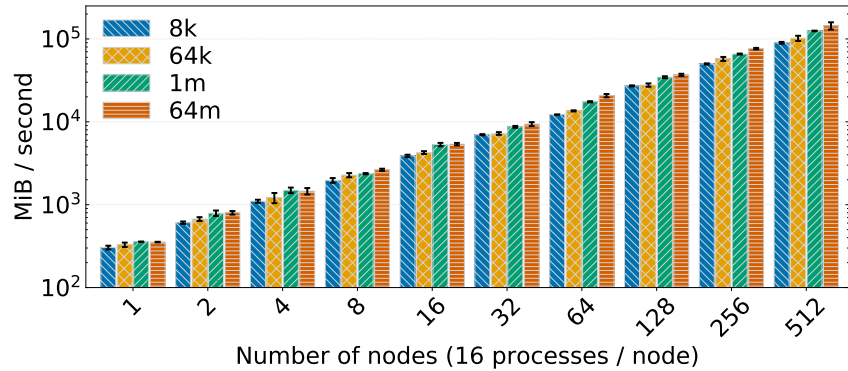

(a) Write throughput

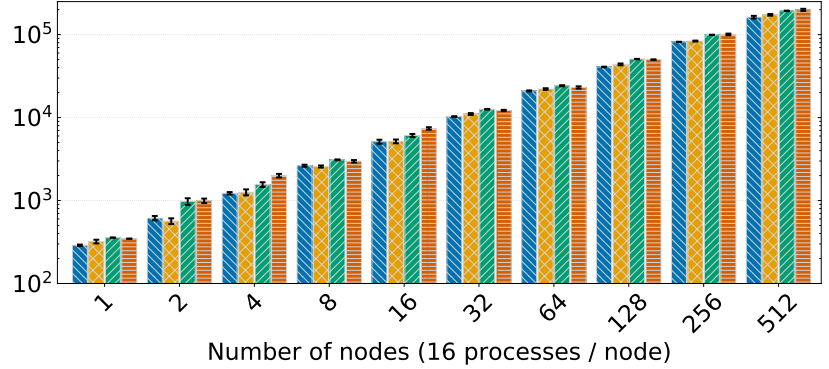

(b) Read throughput

Fig.11. GekkoFS' sequential throughput with a metadata client cache for all processes operating on a single shared file.

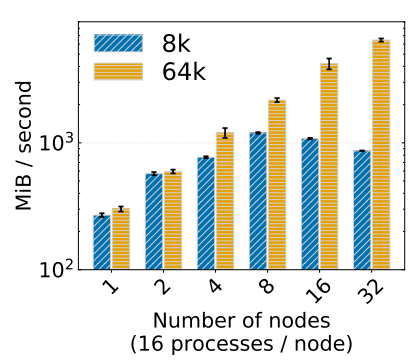

(a) Write throughput

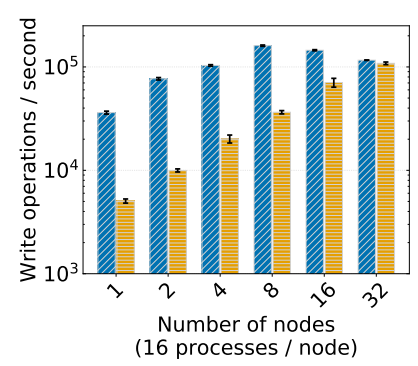

(b) Read throughput
Fig. 12. GekkoFS' sequential write throughput and write IOPS for each process operating on a single shared file.

is shared among many processes or accessed by just a single process. If two processes try to access the same offset in the same chunk file, locking mechanisms of the node-local file system serialize the access to this file.

Figure 12 presents the write throughput and write IOPS for $64 \mathrm{KiB}$ and $8 \mathrm{KiB}$ transfer sizes for a sequentially written single shared file for up to 32 nodes. In these examples a drawback of GekkoFS' synchronous and cache-less design becomes visible and no more than approximately $150 \mathrm{~K}$ write operations per second were achieved. We omit experiments for more than 32 nodes as throughput and IOPS stagnate. This behavior is caused by the size of a single metadata entry that is maintained by only a single node and needs to be updated constantly by all nodes in a mutually exclusive way.

This bottleneck becomes worse for more processes participating in an experiment - eventually reaching a bottleneck, visible at the number of write IOPS. Note that each node in the previous file-per-process experiments only handled at most $25 \mathrm{~K}$ write operations per second on average. Therefore, such behavior did not appear during the previous experiments. The root-cause lies within the number of small RPC messages updating the file size for each write operation, causing network contention on the metadata maintaining node. This observation is supported by the fact that read operations did not show a similar behavior as no metadata updates are necessary and that increasing the number of RPC handler threads did not result in a higher number of write IOPS.

To reduce the number of RPC messages being sent to a single node, we added a rudimentary client cache to locally buffer size updates of a number of write operations before they are send to the node that manages the size of the file. Figure 11 shows the resulting throughput for the cache case for sequential accesses on a single shared file for up to 512 nodes, this time showing close to linear scalability. Due to the internal similarities in data distribution, shared file performances are now comparable to the previously discussed file-per-process performances. While the usage of such a cache may not be ideal for all applications, it significantly prevents node contention. 


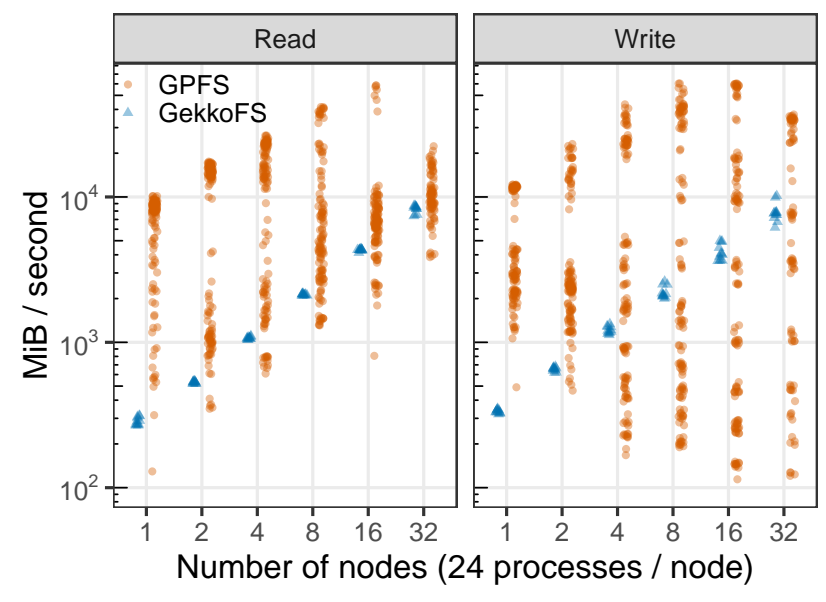

Fig.13. I/O variability of GPFS and GekkoFS of multiple IOR runs on different times and node allocations throughout one week.

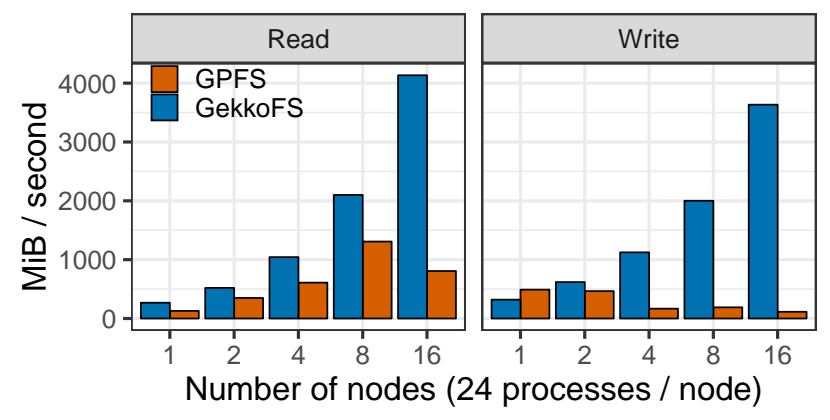

Fig.14. I/O worst-case of GPFS and GekkoFS of multiple IOR runs on different times and node allocations throughout one week.

\subsection{I/O variability and worst-case}

On MareNostrum IV, we used the IOR benchmark to measure the I/O variability and worst-case I/O performance, which applications experience when using GekkoFS compared to the cluster's GPFS. All experiments were run during production, and they were colocated with the ordinary HPC workload. For each file system, we ran 25 independent repetitions of the same benchmark set with each using different node allocations at different times throughout one week.

Each benchmark set used varying I/O request sizes ranging between 512 bytes and $64 \mathrm{MiB}$ to evaluate a more realistic representation of real applications whose I/O request sizes usually differ during execution. GekkoFS chunk size was varied as well, ranging between $128 \mathrm{KiB}$ and $64 \mathrm{MiB}$. GPFS' block size is not dynamically changeable and remained $8 \mathrm{MiB}$. The benchmarks used 24 out of the 48 available cores on each node, and the written file sizes were set large enough to fill the node's main memory to avoid cache effects. The files IOR writes data to and reads from are initially created with each process working on a its own file.

Figure 13 presents the I/O variability of GPFS and GekkoFS as squares and triangles, respectively, up to 32 nodes for each IOR run. GPFS' measured throughput shows significant variability, often scattered by orders of magnitude, with writes showing higher variability than reads. In fact, such I/O behavior is not uncommon at many HPC sites $[42,43,44]$, and it is generally known as the so-called cross-application interference. Cross-application interference is caused by an I/O bottleneck where a shared resource, such as a PFS, is accessed by multiple, uncoordinated applications. With this I/O bottleneck already being a great challenge at many HPC sites today, some studies suggest this issue could become one of the core challenges for Exascale machines in the future $[45,46,47]$.

GekkoFS, on the other hand, shows steady and predictable performance with low cross-application interference, showing the benefits of a private, job-exclusive file system that is accessed by a single application. In both read and write cases, GekkoFS shows close to linear scalability as the available bandwidths and storage capabilities increase with the number of nodes, eventually closing the gap to GPFS' best-case performance. Nonetheless, in terms of the worst-case performance both file systems experience, GekkoFS considerably excels GPFS' throughput in most cases due to the low impact of cross-application interference on GekkoFS, visualized in Figure 14. 


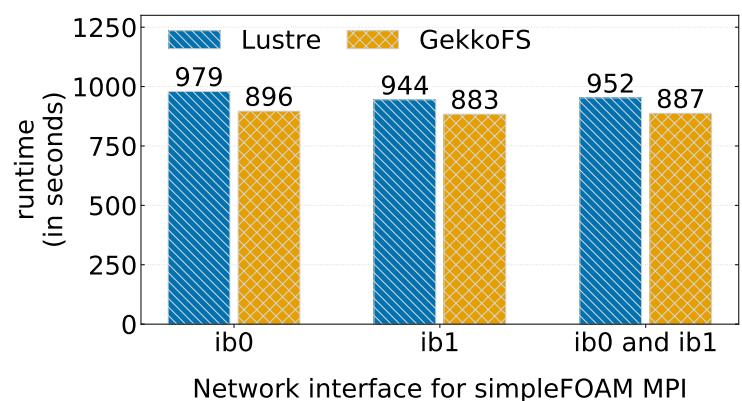

Fig.15. Runtime of the simpleFOAM application when its MPI communication is run on different network interfaces.

\subsection{Effects on the network}

On the NEXTGenIO prototype, we used the user application OpenFOAM to investigate GekkoFS' effects on the network. OpenFOAM [18] is a $\mathrm{C}++$ library for developing user-customized numerical solvers for the solution of Continuum Mechanics problems, including Computational Fluid Dynamics. OpenFOAM solvers often require multiple stages to complete, involve large amounts of $\mathrm{I} / \mathrm{O}$, and benefit from using multiple nodes while parallelization is achieved with MPI. The application is used in industry as well as academia in large scale computations.

In our experiments, we used the simpleFOAM solver which is a steady-state solver for incompressible, turbulent flow, using the SIMPLE (Semi-Implicit Method for Pressure Linked Equations) algorithm. We used 100 iterations with a time step 1 setting which generates around $25 \mathrm{GiB}$ of data in over 170,000 files. The experiments were run on 4 nodes ( 24 processes per node) with simpleFOAM pinned to socket 0. GekkoFS and Lustre both use the ibo Omni-Path adapter. SimpleFOAM's MPI communication was run in three configurations: MPI using the ib0, ib1, or ib0 and ib1 Omni-Path adapters. Hence, ib1 separates MPI from Lustre's and GekkoFS' internal file system network traffic.

Figure 15 shows the runtime (in seconds) of the simpleFOAM experiments when used with Lustre or
GekkoFS. The three bar groups depicts the abovedescribed simpleFOAM MPI configurations for each file system. Although simpleFOAM in this configuration does not generate significant I/O, GekkoFS is still $7 \%$ to $\sim 9 \%$ faster in all cases compared to Lustre. Further profiling revealed that the performance improvements are caused by MPI_Waitall and MPI_Allreduce, instead of from direct file system operations. As a result, both MPI functions potentially benefit from less generated network pollution of GekkoFS. This is because GekkoFS is writing parts of the data locally (see Section 3.6) with less file system traffic being put onto the network in general compared to Lustre, where all file system communication is remote. Therefore, in addition to GekkoFS' linear scaling for metadata and data operations (see Sections 4.2 and 4.3), GekkoFS can also be beneficial for an application's inter-node communication by reducing file system network pollution.

\section{Conclusion}

Increasingly more powerful HPC clusters will lead to even more I/O pressure on a general-purpose PFS, as more applications will concurrently access the shared storage. At the same time, applications with access to node-local SSDs at the compute nodes can use burst buffer file systems to benefit from low-latencies and modern network fabrics, reducing the load on the global PFS. Yet, burst buffer file systems often offer features that scientific applications do not require when running in isolation in an exclusive file system environment.

We have introduced and evaluated GekkoFS, a new burst buffer file system with relaxed POSIX-semantics, which is optimized for access patterns of HPC applications that are known to not work well on a traditional PFS. The POSIX relaxation allows GekkoFS to especially scale metadata operations for accesses to a single directory or even to a single file. We have evalu- 
ated GekkoFS' advantages for typical metadata workloads and have shown that it is able to achieve millions of metadata operations already for a small number of nodes. Moreover, we have presented its linear scalability and low impact of cross-application interference on the file system. We have discussed challenges that occur for shared file I/O operations and how client caches can overcome resulting bottlenecks. Finally, we evaluated GekkoFS' I/O variablity and its effects on the network with the OpenFOAM application.

We plan to extend GekkoFS into three directions. First, we plan an extensive survey on use cases which might benefit from even smaller chunk sizes than the investigated $512 \mathrm{KiB}$, including metadata effects on nodelocal file systems. Second, we plan to investigate how applications can benefit from caching without compromising GekkoFS' lightweight design. Third, we aim to carefully explore how other applications and the file system itself interferes with GekkoFS in more detail to further improve GekkoFS' predictable performance.

\section{Acknowledgments}

We greatly appreciate Franz-Joseph Pfreundt with Fraunhofer ITWM for his assistance on configuring and running BeeGFS BeeOND.

This work has been funded by the German Research Foundation (DFG) through the Priority Programme 1648 "Software for Exascale Computing" and the ADAFS project. It is also partially supported by the Spanish Ministry of Science and Innovation under the TIN201565316 grant, the Generalitat de Catalunya under contract 2014-SGR-1051, as well as the European Union's Horizon 2020 Research and Innovation Programme, under Grant Agreement no. 671951 (NEXTGenIO).

This research was conducted using the supercomputer Mogon II and services offered by Johannes Gutenberg University Mainz. The authors gratefully acknowl- edge the computing time granted on Mogon II.

\section{References}

[1] T. Hey, S. Tansley, and K. M. Tolle, Eds., The Fourth Paradigm: Data-Intensive Scientific Discovery. Microsoft Research, 2009.

[2] R. Ross, R. Thakur, and A. Choudhary, "Achievements and challenges for i/o in computational science," in Journal of Physics: Conference Series, vol. 16 , no. 1,2005 , p. 501.

[3] N. Nieuwejaar, D. Kotz, A. Purakayastha, C. S. Ellis, and M. L. Best, "File-access characteristics of parallel scientific workloads," IEEE Trans. Parallel Distrib. Syst., vol. 7, no. 10, pp. 1075-1089, 1996.

[4] F. Wang, Q. Xin, B. Hong, S. A. Brandt, E. Miller, D. Long, and T. McLarty, "File system workload analysis for large scale scientific computing applications," Lawrence Livermore National Laboratory (LLNL), Livermore, CA, Tech. Rep., 2004.

[5] P. Crandall, R. A. Aydt, A. A. Chien, and D. A. Reed, "Input/output characteristics of scalable parallel applications," in Proceedings Supercomputing '95, San Diego, CA, USA, December 4-8, 1995, 1995, p. 59 .

[6] M. Dorier, G. Antoniu, R. B. Ross, D. Kimpe, and S. Ibrahim, "Calciom: Mitigating I/O interference in HPC systems through cross-application coordination," in 2014 IEEE 28th International Parallel and Distributed Processing Symposium, Phoenix, AZ, USA, May 19-23, 2014, 2014, pp. 155-164.

[7] S. Thapaliya, P. Bangalore, J. F. Lofstead, K. Mohror, and A. Moody, "Managing I/O interference in a shared burst buffer system," in 45th 
International Conference on Parallel Processing, ICPP 2016, Philadelphia, PA, USA, August 1619, 2016, 2016, pp. 416-425.

[8] J. F. Lofstead, S. Klasky, K. Schwan, N. Podhorszki, and C. Jin, "Flexible IO and integration for scientific codes through the adaptable IO system (ADIOS)," in 6th International Workshop on Challenges of Large Applications in Distributed Environments, CLADE@HPDC 2008, Boston, MA, USA, June 23, 2008, 2008, pp. 1524.

[9] M. Folk, A. Cheng, and K. Yates, "Hdf5: A file format and i/o library for high performance computing applications," in Proceedings of supercomputing, vol. 99, 1999, pp. 5-33.

[10] N. Liu, J. Cope, P. H. Carns, C. D. Carothers, R. B. Ross, G. Grider, A. Crume, and C. Maltzahn, "On the role of burst buffers in leadership-class storage systems," in IEEE 28th Symposium on Mass Storage Systems and Technologies, MSST 2012, April 16-20, 2012, Asilomar Conference Grounds, Pacific Grove, CA, USA, 2012, pp. 111.

[11] T. Wang, K. Mohror, A. Moody, K. Sato, and W. Yu, "An ephemeral burst-buffer file system for scientific applications," in Proceedings of the International Conference for High Performance Computing, Networking, Storage and Analysis, SC 2016, Salt Lake City, UT, USA, November 13-18, 2016, 2016, pp. 807-818.

[12] J. Bent, G. A. Gibson, G. Grider, B. McClelland, P. Nowoczynski, J. Nunez, M. Polte, and M. Wingate, "PLFS: a checkpoint filesystem for parallel applications," in Proceedings of the ACM/IEEE Conference on High Performance
Computing (SC), November 14-20, Portland, Oregon, USA, 2009.

[13] M. Vilayannur, P. Nath, and A. Sivasubramaniam, "Providing tunable consistency for a parallel file store," in Proceedings of the FAST '05 Conference on File and Storage Technologies, December 13-16, 2005, San Francisco, California, USA, 2005.

[14] P. H. Lensing, T. Cortes, J. Hughes, and A. Brinkmann, "File system scalability with highly decentralized metadata on independent storage devices," in IEEE/ACM 16th International Symposium on Cluster, Cloud and Grid Computing (CCGrid), Cartagena, Colombia, May 16-19, 2016, pp. $366-375$.

[15] J. Soumagne, D. Kimpe, J. A. Zounmevo, M. Chaarawi, Q. Koziol, A. Afsahi, and R. B. Ross, "Mercury: Enabling remote procedure call for high-performance computing," in 2013 IEEE International Conference on Cluster Computing, CLUSTER 2013, Indianapolis, IN, USA, September 23-27, 2013, 2013, pp. 1-8.

[16] S. Seo, A. Amer, P. Balaji, C. Bordage, G. Bosilca, A. Brooks, P. H. Carns, A. Castelló, D. Genet, T. Hérault, S. Iwasaki, P. Jindal, L. V. Kalé, S. Krishnamoorthy, J. Lifflander, H. Lu, E. Meneses, M. Snir, Y. Sun, K. Taura, and P. H. Beckman, "Argobots: A lightweight low-level threading and tasking framework," IEEE Trans. Parallel Distrib. Syst., vol. 29, no. 3, pp. 512-526, 2018.

[17] P. H. Carns, J. Jenkins, C. D. Cranor, S. Atchley, S. Seo, S. Snyder, and R. B. Ross, "Enabling NVM for data-intensive scientific services," in 4 th Workshop on Interactions of NVM/Flash with Operating Systems and Workloads, INFLOW@OSDI 
2016, Savannah, GA, USA, November 1, 2016., 2016.

[18] H. Jasak, A. Jemcov, Z. Tukovic et al., "Openfoam: A c ++ library for complex physics simulations," in International workshop on coupled methods in numerical dynamics, vol. 1000, 2007, pp. $1-20$.

[19] M. Vef, N. Moti, T. Süß, T. Tocci, R. Nou, A. Miranda, T. Cortes, and A. Brinkmann, "Gekkofs A temporary distributed file system for HPC applications," in IEEE International Conference on Cluster Computing, CLUSTER 2018, Belfast, UK, September 10-13, 2018, 2018, pp. 319-324.

[20] F. B. Schmuck and R. L. Haskin, "GPFS: A shared-disk file system for large computing clusters," in Proceedings of the FAST '02 Conference on File and Storage Technologies, January 28-30, Monterey, California, USA, 2002, pp. 231-244.

[21] P. J. Braam and P. Schwan, "Lustre: The intergalactic file system," in Ottawa Linux Symposium, 2002, p. 50 .

[22] Y. Qian, X. Li, S. Ihara, L. Zeng, J. Kaiser, T. Süß, and A. Brinkmann, "A configurable rule based classful token bucket filter network request scheduler for the lustre file system," in Proceedings of the International Conference for High Performance Computing, Networking, Storage and Analysis (SC), Denver, CO, USA, November 12 - 17, 2017, pp. 6:1-6:12.

[23] F. Herold, S. Breuner, and J. Heichler, "An introduction to beegfs," 2014 , https://www.beegfs.io/docs/whitepapers/ Introduction_to_BeeGFS_by_ThinkParQ.pdf.
[24] R. B. Ross and R. Latham, "PVFS - PVFS: a parallel file system," in Proceedings of the ACM/IEEE SC2006 Conference on High Performance Networking and Computing, November 11-17, 2006, Tampa, FL, USA, 2006, p. 34.

[25] S. Oral and G. Shah, "Spectrum scale enhancements for coral. presentation slides at supercomputing'16." 2016, http: //files.gpfsug.org/presentations/2016/SC16/ 11_Sarp_Oral_Gautam_Shah_Spectrum_Scale_ Enhancements_for_CORAL_v2.pdf.

[26] A. Kougkas, H. Devarajan, and X. Sun, "Hermes: a heterogeneous-aware multi-tiered distributed I/O buffering system," in Proceedings of the 27th International Symposium on HighPerformance Parallel and Distributed Computing, HPDC 2018, Tempe, AZ, USA, June 11-15, 2018, 2018, pp. 219-230.

[27] R. Latham, R. B. Ross, and R. Thakur, "The impact of file systems on MPI-IO scalability," in Recent Advances in Parallel Virtual Machine and Message Passing Interface, 11th European PVM/MPI Users' Group Meeting, Budapest, Hungary, September 19-22, 2004, Proceedings, 2004, pp. 87-96.

[28] A. Choudhary, W.-k. Liao, K. Gao, A. Nisar, R. Ross, R. Thakur, and R. Latham, "Scalable i/o and analytics," in Journal of Physics: Conference Series, vol. 180, no. 1, 2009, p. 012048.

[29] M. Moore, D. Bonnie, B. Ligon, M. Marshall, W. Ligon, N. Mills, E. Quarles, S. Sampson, S. Yang, and B. Wilson, "Orangefs: Advancing pvfs," FAST poster session, 2011. 
[30] D. Ritchie and K. Thompson, "The UNIX timesharing system (reprint)," Commun. ACM, vol. 26, no. 1, pp. $84-89,1983$.

[31] M.-A. Vef, V. Tarasov, D. Hildebrand, and A. Brinkmann, "Challenges and solutions for tracing storage systems: A case study with spectrum scale," ACM Trans. Storage, vol. 14, no. 2, pp. 18:1-18:24, 2018.

[32] S. Patil and G. A. Gibson, "Scale and concurrency of GIGA+: file system directories with millions of files," in 9th USENIX Conference on File and Storage Technologies, San Jose, CA, USA, February 15-17, 2011, 2011, pp. 177-190.

[33] K. Ren, Q. Zheng, S. Patil, and G. A. Gibson, "Indexfs: Scaling file system metadata performance with stateless caching and bulk insertion," in International Conference for High Performance Computing, Networking, Storage and Analysis, SC 2014, New Orleans, LA, USA, November 16-21, 2014, 2014, pp. 237-248.

[34] P. Carns, Y. Yao, K. Harms, R. Latham, R. Ross, and K. Antypas, "Production i/o characterization on the cray xe6," in Proceedings of the Cray User Group meeting, vol. 2013, 2013.

[35] J. Xing, J. Xiong, N. Sun, and J. Ma, "Adaptive and scalable metadata management to support a trillion files," in Proceedings of the ACM/IEEE Conference on High Performance Computing, SC 2009, November 14-20, 2009, Portland, Oregon, USA, 2009.

[36] W. Frings, F. Wolf, and V. Petkov, "Scalable massively parallel I/O to task-local files," in Proceedings of the ACM/IEEE Conference on High Performance Computing (SC), November 14-20, Portland, Oregon, USA, 2009.
[37] S. Yang, W. B. Ligon III, and E. C. Quarles, "Scalable distributed directory implementation on orange file system," Proc. IEEE Intl. Wrkshp. Storage Network Architecture and Parallel I/Os (SNAPI), 2011.

[38] S. Patil, K. Ren, and G. Gibson, "A case for scaling HPC metadata performance through despecialization," in 2012 SC Companion: High Performance Computing, Networking Storage and Analysis, Salt Lake City, UT, USA, November 1016, 2012, 2012, pp. 30-35.

[39] P. H. Carns, W. B. L. III, R. B. Ross, and R. Thakur, "PVFS: A parallel file system for linux clusters," in 4th Annual Linux Showcase 8 Conference 2000, Atlanta, Georgia, USA, October 10-14, 2000, 2000.

[40] S. Dong, M. Callaghan, L. Galanis, D. Borthakur, T. Savor, and M. Strum, "Optimizing space amplification in rocksdb," in CIDR 2017, 8th Biennial Conference on Innovative Data Systems Research, Chaminade, CA, USA, January 8-11, 2017, Online Proceedings, 2017.

[41] S. Oral, D. A. Dillow, D. Fuller, J. Hill, D. Leverman, S. S. Vazhkudai, F. Wang, Y. Kim, J. Rogers, J. Simmons et al., "Olcfs $1 \mathrm{tb} / \mathrm{s}$, next-generation lustre file system," in Proceedings of Cray User Group Conference (CUG 2013), 2013, pp. 1-12.

[42] J. F. Lofstead, F. Zheng, Q. Liu, S. Klasky, R. Oldfield, T. Kordenbrock, K. Schwan, and M. Wolf, "Managing variability in the IO performance of petascale storage systems," in Conference on High Performance Computing Networking, Storage and Analysis,SC 2010, New Orleans, LA, USA, November 13-19, 2010, 2010, pp. 1-12. 
[43] B. Xie, J. S. Chase, D. Dillow, O. Drokin, S. Klasky, S. Oral, and N. Podhorszki, "Characterizing output bottlenecks in a supercomputer," in SC Conference on High Performance Computing Networking, Storage and Analysis, SC '12, Salt Lake City, UT, USA - November 11 - 15, 2012, 2012, p. 8.

[44] A. Kougkas, H. Devarajan, X. Sun, and J. F. Lofstead, "Harmonia: An interference-aware dynamic I/O scheduler for shared non-volatile burst buffers," in IEEE International Conference on Cluster Computing, CLUSTER 2018, Belfast, UK, September 10-13, 2018, 2018, pp. 290-301.

[45] Y. Hashimoto and K. Aida, "Evaluation of performance degradation in HPC applications with VM consolidation," in Third International Conference on Networking and Computing, ICNC 2012, Okinawa, Japan, December 5-7, 2012, 2012, pp. 273277.

[46] J. F. Lofstead and R. Ross, "Insights for exascale IO apis from building a petascale IO API," in International Conference for High Performance Computing, Networking, Storage and Analysis, SC'13, Denver, CO, USA - November 17 - 21, 2013, 2013, pp. 87:1-87:12.

[47] D. A. Reed and J. J. Dongarra, "Exascale computing and big data," Commun. ACM, vol. 58, no. 7, pp. 56-68, 2015.

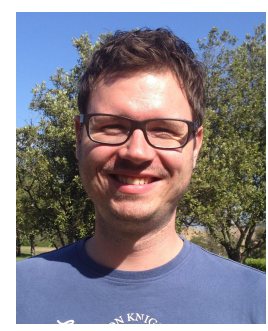

Marc-André Vef is a thirdyear Ph.D. candidate in André Brinkmann's research team at the Johannes Gutenberg University Mainz. He started his Ph.D. in 2016 after receiving his B.Sc. and M.Sc. degrees in computer science from the Johannes Gutenberg University Mainz. His master thesis was in cooperation with IBM Research about analyzing file create performance in the IBM Spectrum Scale parallel file system (formerly GPFS). Marc's research interests focus on parallel and ad-hoc file systems and system analytics.

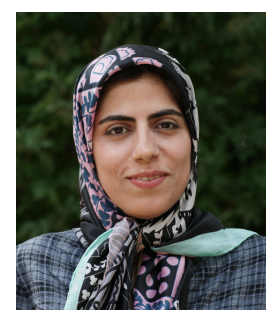

Nafiseh Moti is a Ph.D. student in Computer Science at the Johannes Gutenberg University of Mainz. Currently, she is a research assistant at Efficient Computing and Storage Group at Center for Data Processing at the University of Mainz. Her main research interests include: User-level File Systems and File Systems and Data Structures for Storage Class Memories.

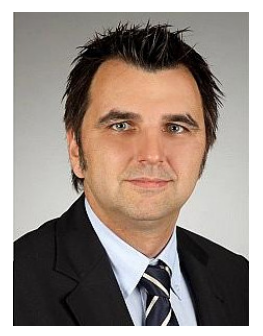

Tim Süß is a full professor at the computer science department of the Fulda University of Applied Science (since 2019). He received his Ph.D. in computer science in 2011 from the University of Paderborn and has been post-doc at the Johannes Gutenberg University Mainz from 2012 to 2019 with an intermission as temporal professor for Applied Computer Science from 2017 to 2018. His research interests focus on techniques for accelerating parallel computing, effective parallel scheduling, and storage systems.

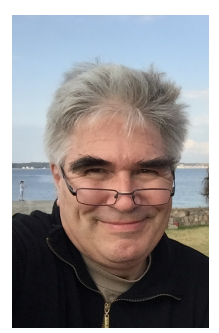

Markus Tacke is the technical head of HPC (since 2015) at the center for data processing (ZDV) at the Johannes Gutenberg University, Mainz, Germany. He studied Mathematic (and Astronomy) at the WWU Münster, Germany where he received his M.Sc. in 1987 in the field of applied Mathematics. He joined the ZDV in 1991 and has been in charge of several responsibilities, including the design of the university's two supercomputers (since 1994). However, his true passion lies in parallel file systems. 
Tommaso Tocci graduated in computer science at Sapienza University of Rome and he concluded his Master's degree in Distributed Systems at Universitat Politècnica de Catalunya (UPC). At the moment is employed at Barcelona Supercomputing Center (BSC), investigating new distributed storage solutions for the upcoming exascale computing era.

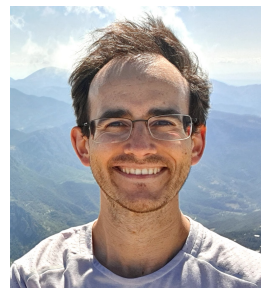

Ramon Nou has been working at BSC on the Autonomic System and e-Business Platforms group of the Barcelona Supercomputing Center (BSC) until 2008 when he switched to the Storage-system group at BSC since 2009 as a researcher. In 2008, he obtained his Ph.D. with the topic "Using online simulation to improve QoS on middleware". Ramon has a wide view on all computer levels, with expertise in optimization, performance measurements and simulation/modelling of complex systems. Now is co-leader of the Storage Systems for Extreme Computing research group since January 2019.

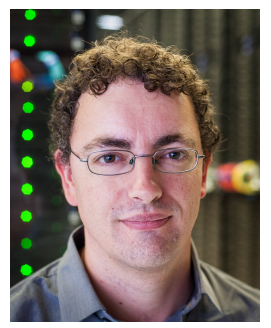

Alberto Miranda is a Senior Researcher in advanced storage systems in the Computer Science Department of the Barcelona Supercomputing Center (BSC) and co-leader of the Storage Systems for Extreme Computing research group since January 2019. His research interests include scalable storage technologies, architectures for distributed systems, operating system internals, and high performance networking. He received a Ph.D. Cum Laude in Computer Science from the Technical University of Catalonia (UPC) in 2014, and has been working at BSC since 2007.

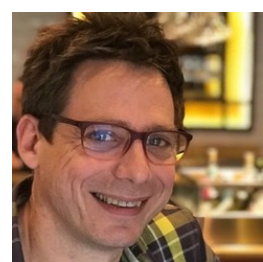

Toni Cortes is an associate professor at Universitat Politècnica de Catalunya (since 1998) and researcher at the Barcelona Supercomputing Center. He received his M.S. in computer science in 1992 and his Ph.D. also in computer science in 1997 (both at Universitat Politècnica de Catalunya). Currently he develops his research at the Barcelona Supercomputing Center, where he acted as the leader of the Storage Systems Research Group from 2006 until 2019. His research concentrates in storage systems, programming models for scalable distributed systems and operating systems. He is also editor of the Cluster Computing Journal and served as the coordinator of the SSI task in the IEEE TCSS. He has also served in many international conference program committees and/or organizing committees and was general chair for the Cluster 2006 and 2021 conference, LaSCo 2008, XtreemOS summit 2009, and SNAPI 2010. He is also served as the chair of the steering committee for the Cluster conference series (2011-2014). His involvement in IEEE CS has been awarded by the "Certificate of appreciation" in 2007.

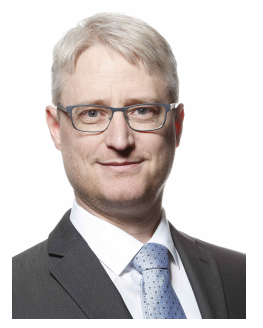

André Brinkmann is a full professor at the computer science department of JGU and head of the university's data center ZDV (since 2011). He received his Ph.D. in electrical engineering in 2004 from the Paderborn University and has been an assistant professor in the computer science department of the Paderborn University from 2008 to 2011. Furthermore, he has been the managing director of the Paderborn Centre for Parallel Computing $\mathrm{PC}^{2}$ during this time frame. His research interests focus on the application of algorithm engineering techniques in the area of data centre management, cloud computing, and storage systems. 